\title{
Effect of Suprathermal Electrons on Central Plasma Relaxation Oscillations During Localized Electron Cyclotron Heating on the HL-1M Tokamak
}

\author{
Y. Liu, G.C. Guo, X.T. Ding, and K.L. Wong* \\ Southwestern Institute of Physics, Chengdu, Sichuan 610041, People's Republic of China \\ * Plasma Physics Laboratory, Princeton University, Princeton, New Jersey 08543, U.S.A
}

Received on 26 June, 2001

\begin{abstract}
In this paper we discuss the typical behavior of HL-1M tokamak plasmas during ECRH. A large variety of sawtooth types have been observed at different ECH power deposition locations. The observed central MHD activities detected by the soft x-ray diode array include saturated sawtooth, partially saturated sawtooth, double sawtooth, and strong $m=1$ bursts superimposed on the ramp phase of sawtooth. Complete suppression of sawtooth is achieved during ECRH, when the heating power is applied on the high-field side of low-density plasmas, and exceeds a threshold value of power. The $m=1$ bursts riding on the ramp phase of sawtooth can only be excited when the ECRH location is near the $\mathrm{q}=1$ surface at the high field side. The conditions under which the various relaxation activities are produced, or suppressed, are described. Experimental results imply that the energetic electrons, generated during ECRH, are responsible for the modification or stabilization or excitation of the instability. Near the $q=1$ surface, while the passing electrons play a role to reduce the shear and tend to stabilize the sawtooth activity, the barely-trapped electrons play a role to enhance or drive an internal kink instability.
\end{abstract}

\section{Introduction}

Sawtooth oscillations in tokamaks have long been the subject of intense study, and significative efforts have been carried out to control this instability. It has been recognized that sawtooth oscillations can be stabilized by electron-cyclotron-resonance heating $(\mathrm{ECH})$, and a variety of non-standard sawtooth activities has been observed during ECH. In DIII tokamak [1], sawtooth oscillation with two periods and amplitudes, per cycle, have been observed, and non-standard sawtooth (saturated and partial saturated sawtooth) have been reported in WT- $3^{2}$ and $\mathrm{TCV}^{3}$ experiments. Recently, excitation of internal kink instability during off-axis ECH and neutral beam injection was reported in DIII-D tokamak [4]. These different relaxations have been observed on HL-1M tokamak under different heating condition. In HL-1M experiments, in addition to sawtooth activity, the $\mathrm{m}=1$ modes are observed to be strongly modified (preceding the crash) and /or excited (riding on the ramp phase) by ECH. The sharply localized nature of the ECH provides an effective tool for modifying plasma parameters locally near the $\mathrm{q}=1$ surface. These observations, as well as plausible theoretical explanation for the stabilization, are the subject of this paper.

\section{Experimental set-up}

The HL-1M tokamak has circular cross section with major radius $\mathrm{R}=1.02 \mathrm{~m}$, minor radius $\mathrm{a}=0.26 \mathrm{~m}$, lineaveraged electron density $\bar{n}_{e}=(0.3 \sim 2) \times 10^{13} \mathrm{~cm}^{-3}$ central electron temperature $T_{e}(0) \approx 1 \mathrm{Kev}$, central ion temperature $T_{i}(0) \approx 400 \mathrm{eV}$, plasma current $I_{p}$ in the range of $160 \mathrm{kA}$ to $200 \mathrm{kA}$, and toroidal magnetic field in the range of $2.4 \mathrm{~T}$ to $2.8 \mathrm{~T}$ at $\mathrm{R}=1.02 \mathrm{~m}$. A 75 $\mathrm{GHz}$ gyrotron powers the ECH system up to $400 \mathrm{~kW}$ for fundamental electron cyclotron resonance (ECR) at $\mathrm{B}=2.68 \mathrm{~T}$. The ECH pulse lengths are in the range of $30-50$ msec. Electron cyclotron waves, polarized at the ordinary mode, are launched perpendicular to the toroidal magnetic field from the low field side of the torus. The fundamental electron cyclotron resonance (ECR) location is changed in the range from low field side to high-field side to scan the MHD response.

A 60-channel soft x-ray detector array placed at the top of the tokamak was used to monitor the plasma MHD activity. The system covers the entire plasma cross section through a $12.5 \mu \mathrm{m}$ thick beryllium foil; only those channels with sight lines passing through the plasma core are shown in this work because our attention is focused at the $q=1$ surface in the plasma core. With zero bias voltage, the time response of the 
detector-preamplifier system was $100 \mathrm{~Hz}$, and radial resolution was $2 \mathrm{~cm}$. The soft $\mathrm{x}$-ray system allows us to follow the fast temporal dynamics of various MHD phenomena, especially sawtooth events (with a shortest repetition time of $2 \mathrm{~ms}$ ), and the $(1,1)$ MHD mode (with a rotational period of $0.1 \mathrm{~ms}$ ).

\section{Effect of ECRH on MHD ac- tivities}

\section{III.1 Characteristics of sawteeth types during ECRH}

The sawtooth activity was observed to be strongly modified by localized ECRH near the $q=1$ surface in HL-1M. Experiments were performed when the current was such that the safety factor at the limiter was in the range $q_{L}=4.0-5.0$. Non-standard sawtooth activity, such as double sawtooth, partially saturated and saturated sawtooth, have been observed while changing the heating location, the ECRH power and the electron density.

These modifications of the type of sawtooth are very sensitive to the field $B_{T}$ which determines the location where the ECH is localized. By varying the toroidal magnetic field, the ECR location is changed along the direction of $\mathrm{R}$ from the high-field side to the low-field side. In our analysis we approximate the radius of the $q=1$ surface by the inversion radius of sawtooth (as often noted, $r_{q=1}$ is a little larger than $\left.r_{\text {inv }}\right)$. The geometric configuration is shown in Fig.1 (a) while the changes in the soft-x ray (SXR) signal due to the ECH are shown in Figs.1(b)-(e). When the ECR is at the low-field side there is little affection on the sawtooth activity, as shown in Fig.1 (b). When ECR is applied at the magnetic axis around point c, Fig.1 (c), the amplitudes of the sawtooth oscillation are very large with sharp ramps and deep drops, and there are some double sawtooth interspersing among single sawtooth. When the off-axis heating location is in the range around point $\mathrm{d}$, double sawtooth, which exhibit two rises and two crashes in a cycle, can be observed, as shown in Fig.1 (d). The onset of the first crash caused a drop of $5 \%(\Delta A / A)$ in soft x-ray emission, while a large drop $\Delta A / A \approx 15 \%$ was observed during the second crash (for normal sawteeth $\Delta A / A \approx 10 \%$ ). When the ECR is applied around the $\mathrm{q}=1$ surface, the sawtooth oscillations can be completely stabilized as shown in Fig.1(e). Though the sawtooth suppression was only observed when heating was deposited near the $q=1$ surface, it must be noted that the double sawtooth can be observed in a larger region. (a)

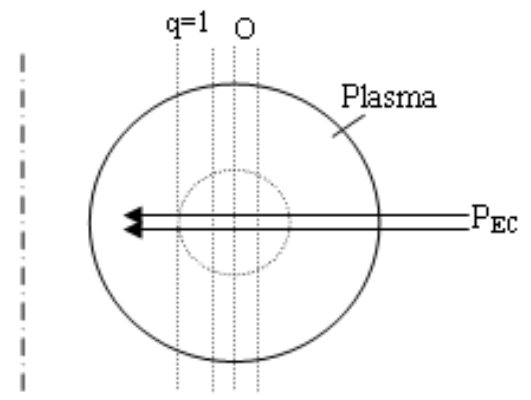

$(e)(d)(c)(b)$

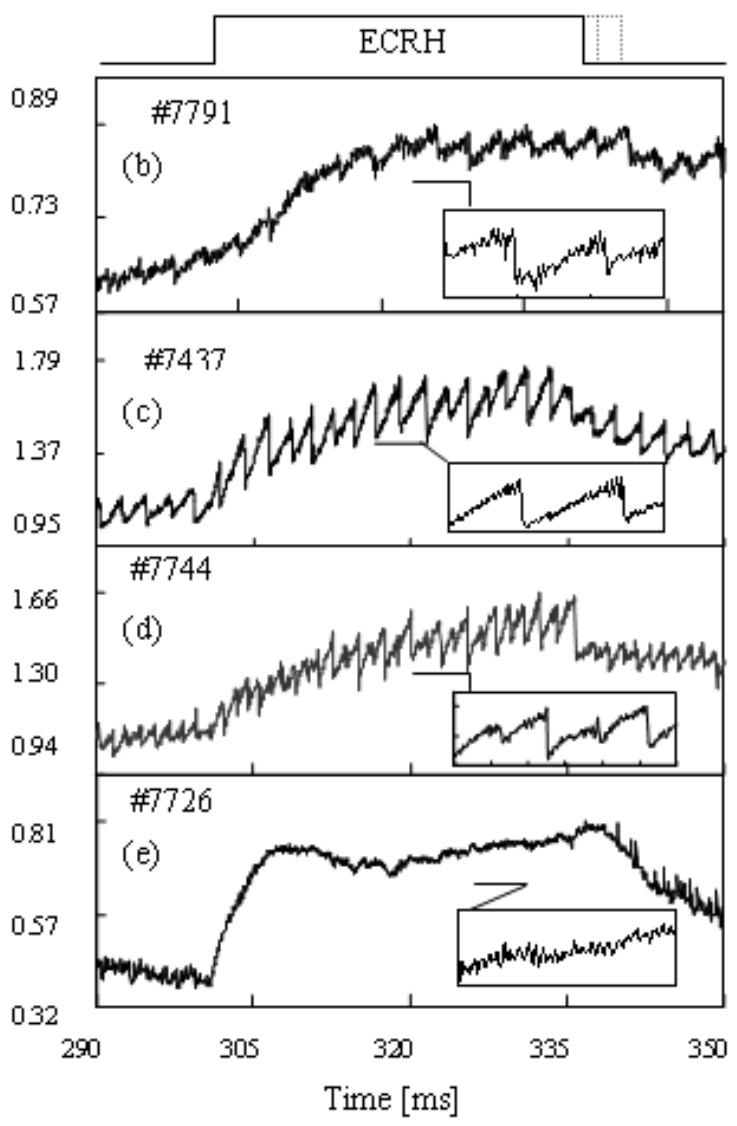

Figure 1. Fig. 1. (a) Schematic diagram showing the $q=1$ surface and the wave trajectory in the experiment. The cyclotron resonance locations are shown as vertical lines for the four cases (b), (c), (d) and (e) shown below. Evolution of the soft x-ray signal for (b) ECH on low-field side $\left(B_{T}=2.75\right)$, (c) ECR on-axis heating $\left(B_{T}=2.65\right)$, (d) ECH on high-field side $\left(B_{T}=2.60\right)$, and (e) ECH at $\mathrm{q}=1$ surface on high-field side $\left(B_{T}=2.54\right)$, for and $q_{L}=4$, $I_{P}=150-200 k A$ and $P_{E C H}=230 k W$

In addition to the influence of location of $\mathrm{ECH}$, other plasma parameters play important roles as well. These parameters include the electron density, the power of ECH and the plasma current. The points in Fig. 2 stands for a set of off-axis ECR heating discharges in which parameter scans were carried out. It is clear from this figure that different MHD relaxation activities occur in different parameter regions. Double sawtooth activity is noticeable especially at high 
ECRH power and medium density $\left(\bar{n}_{e} \approx 1.6 \times 10^{13}\right)$ $\left.\mathrm{cm}^{-3}\right)$, with high plasma current $\left(I_{P} \approx 200 \mathrm{kA}\right)$. In contrast, at low ECRH power, instead of change of the shape of sawteeth, strong bursts of $m=1$ mode were frequently observed in the region of medium density, which will be discussed in III.2. In the region near the $q=1$ surface, the complete stabilization of sawtooth can only be obtained when electron density is low enough $\left.\left(\bar{n}_{e} \approx 0.6 \times 10^{13}\right) \mathrm{cm}^{-3}\right)$. Above this value, but still in the region of low density $\left.\left(\leq \bar{n}_{e} \approx 1.0 \times 10^{13}\right) \mathrm{cm}^{-3}\right)$, we usually can observe partially saturated or saturated sawtooth according to the deposited ECRH power as shown in Fig 3. The crash of a saturated sawtooth involves a larger amplitude change $(\Delta A / A \approx 15 \%)$, when compared with a single sawtooth, and the energy transport induced by the saturated sawtooth is larger than that due to a single sawtooth of the same cycle length. This implies that during the saturated phase there are much energy and impurity accumulated in the central region.

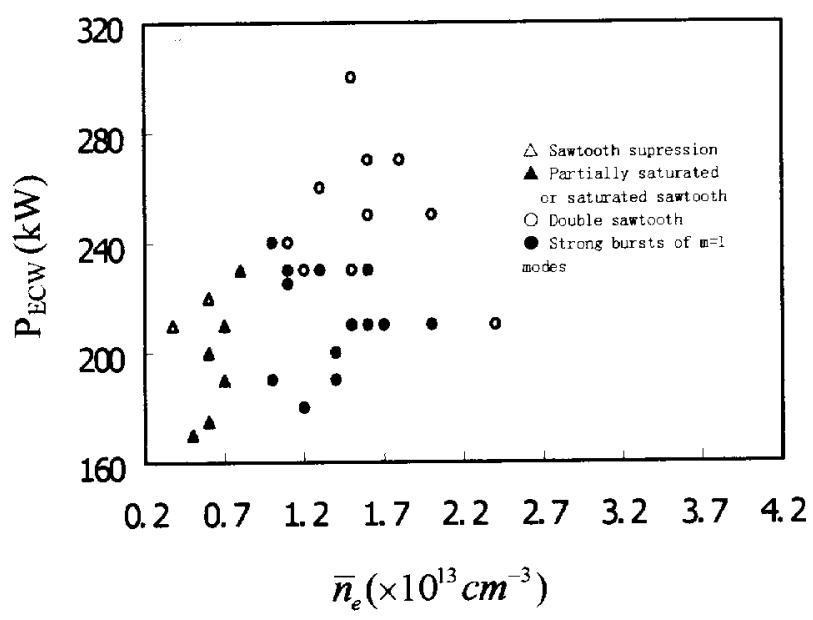

Figure 2. Diagram for off-axis heating, showing the $P_{E C W}-$ $\bar{n}_{e}$ plane. The symbols indicate the onset of MHD activities in various situations. All the data here were got when the ECRH location was near or in small distance off the sawtooth inversion on the high field-side.

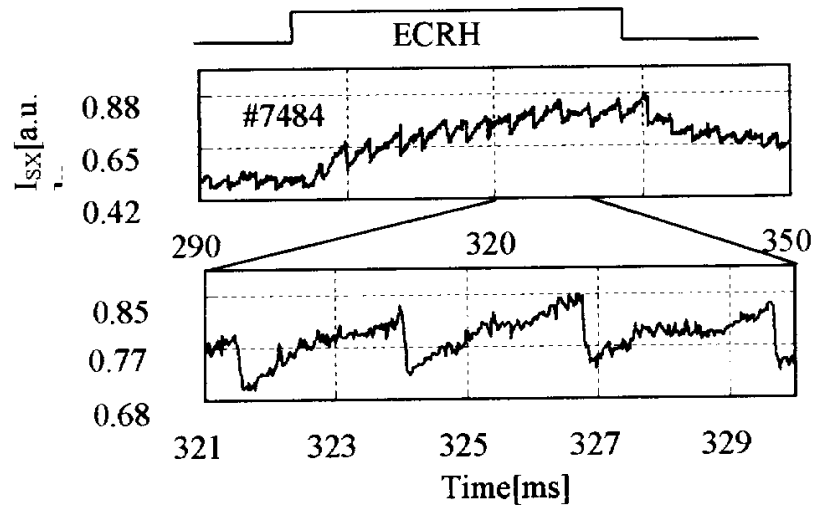

Figure 3(a). Expanded view of partially saturated sawtooth $\left(B_{T}=2.63 \mathrm{~T}, I_{P}=205 \mathrm{kA}, P_{E C}=200 \mathrm{~kW}, \bar{n}_{e}=\right.$ $\left.0.7 \times 10^{13} \mathrm{~cm}^{-3}\right)$.

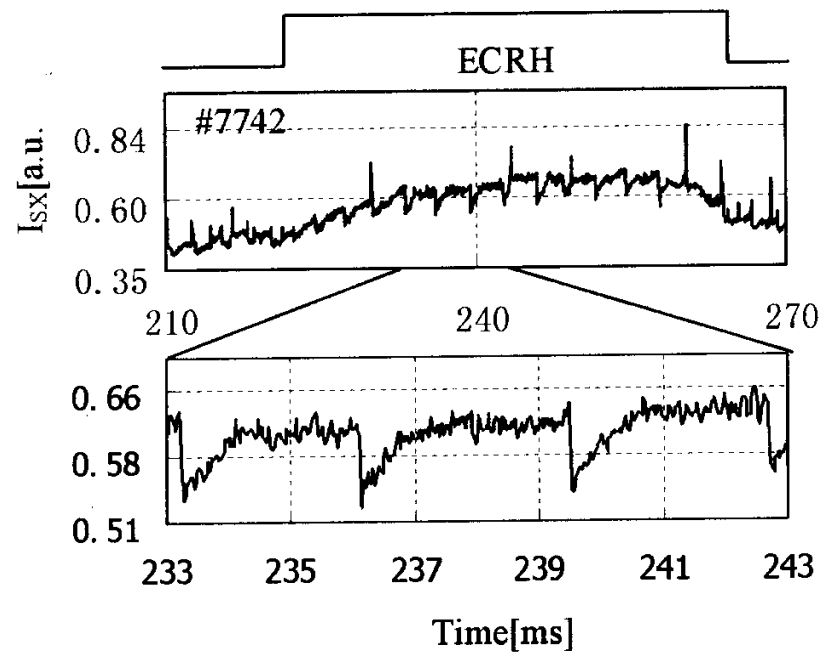

Figure 3(b). Saturated sawtooth activities $\left(B_{T}=2.56 \mathrm{~T}\right.$, $\left.I_{P}=205 \mathrm{kA}, P_{E C}=200 \mathrm{~kW}, \bar{n}_{e}=0.6 \times 10^{13} \mathrm{~cm}^{-3}\right)$.

These observations clearly indicate the critical importance of the location of the application of ECH, and the important role played by the other plasma parameters. With off-axis ECRH, the basic type of nonstandard sawtooth is double sawtooth, while the partially saturated and saturate sawtooth might be formed due to the stabilization of the first sawtooth crash, during the heating. Standard sawtooth are produced by on axis power deposition, and suppression of sawtooth is caused by deposition close to the $q=1$ surface on highfield side, when $n_{e}$ is low enough.

\section{III.2 Internal MHD modes}

With medium heating power applied off axis, near the $q=1$ surface on the medium density $\left(\geq 1.0 \times 10^{13}\right.$ $\mathrm{cm}^{-3}$ ) plasma discharges, the normal structure of sawtooth oscillation does not change much but the modes riding on change. Instead of a single growing envelope of $m=1$ before the crash of the sawtooth, strong bursts of $m=1$ localized near the $q=1$ surface frequently appear during the ramp phase of sawtooth. Fig. 4 shows the sawtooth and $\mathrm{m}=1$ mode with $230 \mathrm{~kW}$ of $\mathrm{ECRH}$ power. Often a clear $\mathrm{m}=1$ precursor is observed prior to the crash, but the precursor amplitude $(\triangle A / A \approx 15 \%)$ is smaller than that of the burst $(\triangle A / A \approx 5 \%)$ that appears earlier. At low ECH power, a burst of the $\mathrm{m}=1$ mode breaks out at earlier time and it merges as to become the precursor of the sawtooth. At high ECRH powers, a burst of $\mathrm{m}=1$ mode appears in the reheat phase of the sawtooth and, sometimes, it separates from the precursor oscillations before the crash, i.e, it looks like one single burst in the typical fishbone activities. 


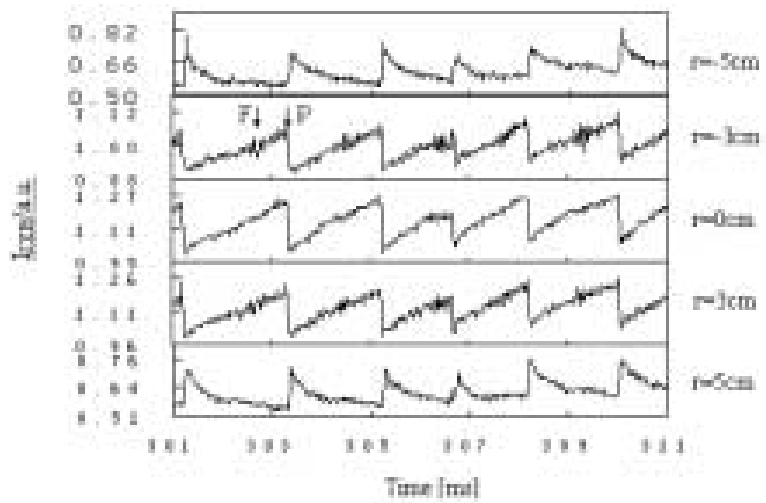

Figure 4. Strong burts of $\mathrm{m}=1$ mode frequently appear when the ECRH locations is near the $\mathrm{q}=1$ surface. Fig. 3(a). Expanded view of partially saturated sawtooth $\left(B_{T}=\right.$ $\left.2.55 \mathrm{~T}, I_{P}=200 \mathrm{kA}, P_{E C}=230 \mathrm{~kW}, \bar{n}_{e} \cong 1.6 \times 10^{13} \mathrm{~cm}^{-3}\right)$. $\mathrm{F}$ - fishbone-like, $\mathrm{P}$ - precursor.

The duration of the burst lasts for a long time $\left(\Delta \tau / \tau_{s} \approx 25 \%\right)$ while the precursor was observable for only a small fraction $\left(\Delta \tau / \tau_{s} \approx 5 \%\right)$ of the sawtooth period. The oscillation frequency within a burst of $\mathrm{m}=1$ is $\sim 8 \mathrm{kHz}$, which is nearly the same as that of the precursors.

In order to investigate the effect of heating location on mode activity, a detailed toroidal field scan was performed at $230 \mathrm{~kW}$ of ECW input power. A summary of the results is displayed in Fig.5. The abscissa of the plots is the central toroidal field. There is a maximum in the amplitude of the burst when the $\mathrm{ECH}$ resonance was placed at $r / a=0.3$. This was close to the surface corresponding to he sawtooth inversion. The effect on the $m=1$ burst was very sensitive to the heating location when it was near the $\mathrm{x}$-ray sawtooth inversion. A shift of $2 \mathrm{~cm}$ in the resonance location changed the relative amplitude by a factor of 2 , from 0.20 to 0.9 . Most of the data points in Fig.5 are taken from discharges with ECW pulses of $30 \mathrm{msec}$ and plasma density $\bar{n}_{e} \cong 1.5 \times 10^{13} \mathrm{~cm}^{-3}$

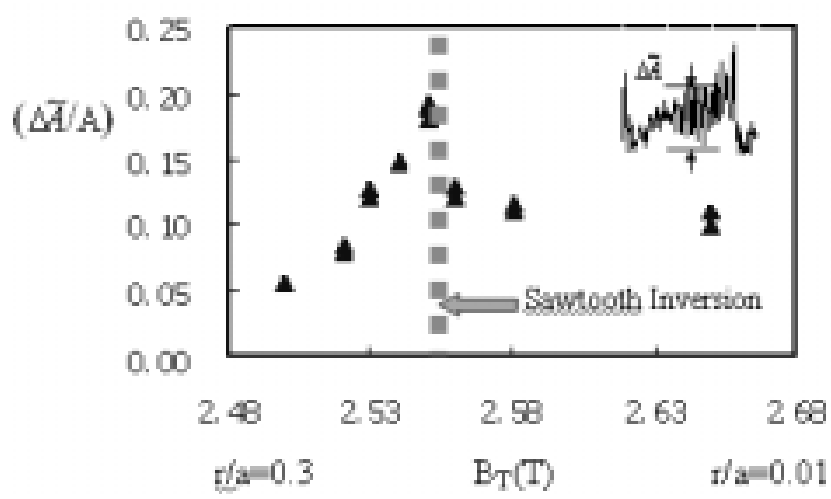

Figure 5. Amplitude of the parasitic $\mathrm{m}=1$ mode with respect to toroidal field, plasma current $I_{p}=180 \mathrm{kAm}$ line averaged density Figure 3(a). Expanded view of partially saturated sawtooth $\bar{n}_{e} \cong 1.5 \times 10^{13} \mathrm{~cm}^{-3}$ and incident ECRH power $P_{0}=230 \mathrm{~kW}$. Here $\Delta A$ is the amplitude of the parasitic $\mathrm{m}=1$ and $A$ is the amplitude of soft x-ray signal.

\section{Discussion}

\section{IV.1 Effect of suprathermal electrons on saw- tooth stabilization}

To understand the condition under which one of the non-standard sawtooth will occur and the mechanism of sawtooth stabilization, it is helpful to examine the role of high-energy electrons produced by ECRH. The x-ray energy spectrum shows a high-energy tail indicating the presence of hot-electron population with $T_{e h} \approx 30 \mathrm{keV}$ extending up to $300 \mathrm{keV}$. In the absence of $\operatorname{ECRH}\left(T_{e} \approx 1 \mathrm{keV}\right)$, hot-electron tails are not observed. In discharges where we obtain sawtooth stabilization or saturated sawtooth, during ECRH, we have often observed sharp spike caused by runaway electron instability on ECE and SXR raw signals. These observations suggest that the high-energy tail electrons generated by ECRH in the low-density regime may be responsible for the modification/stabilization of the MHD behaviour in the plasma center. As we know, the high energetic electrons will be trapped, barely-trapped or circulate in the torus, and the distribution will be different as the ECRH power and position of resonance change. This may lead to the occurrence of various non-standard sawtooth activities.

By applying ECH on the high-field side within $q=1$ surface, the high-energy electrons can traverse most of the surface enhancing a locally peaked current profile on the entire surface. This incremental change of the current density would result in a local reduction of the shear:

$$
s=\frac{r d q}{q d r}
$$

It is known that for most of present-day tokamaks, the central plasma beta value is still lower than the critical one for exciting the ideal internal kink mode [4], implying that the instability for sawtooth activity is the resistive internal kinks. The growth rate of the resistive internal kink mode depends strongly on the magnetic shear $S$ [5]. The mode becomes stable only when shear is smaller than a critical value:

$$
\begin{gathered}
\omega=\frac{\gamma_{I}^{2}}{\omega_{* e}} \\
\frac{\gamma}{\omega_{* i}}=\frac{4}{\pi^{2}}\left(\frac{\omega_{A}^{7}}{\omega_{* i}^{4}\left|\omega_{* e}\right|^{3}}\right) S_{m}^{-1}\left(\frac{\rho_{i}}{r_{1}}\right) S^{4}\left(S^{2}-S_{e}^{2}\right)
\end{gathered}
$$

where,

$$
\begin{gathered}
\gamma_{I}=\left(\frac{3 \pi}{2}\right) \omega_{A}\left(\frac{r_{1}}{R}\right)^{2}\left[\beta_{P}^{2}-\frac{13}{144}\right] \\
S_{m}=\omega_{A} \tau_{R}
\end{gathered}
$$




$$
\begin{gathered}
\tau_{R}=\left(\frac{4 \pi r_{1}^{2}}{\eta c^{2}}\right) \\
\omega_{A}=\frac{B}{\sqrt{12 \pi \rho R}} \\
S_{c}=\omega_{* i}^{1 / 4}\left|\omega_{* e}\right|^{1 / 2} S_{m}^{1 / 4}\left(\frac{\rho_{i}}{r_{1}}\right)^{1 / 2} \omega_{A}^{-5 / 4} \gamma_{I}^{1 / 2} . \\
\omega_{* i}=\frac{c}{Z e B n \gamma_{1}}\left(d P_{1} / d r\right)_{r_{1}}, \\
\omega_{* e}=\frac{c}{e B n r_{1}}\left(\frac{d P_{e}}{d r}\right)_{\eta}+0.71 \frac{c}{e B r_{1}}\left(\frac{d T_{e}}{d r}\right)_{r_{1}}
\end{gathered}
$$

We learn from these result that the unstable mode rotates in the direction of the ion diamagnetic drift, and the growth rate, on the other hand, is proportional to $S$ [6], implying that the instability occurs almost suddenly.

The reduction of shear caused by suprathermal electrons has a stabilizing effect on the mode. If this reduction of shear occurs at the $q=1$ surface, it may stabilize the $m / n=1 / 1$ resistive mode responsible for the sawtooth activity. Since the critical magnetic shear is determined by the electron density, only when the electron density is low enough, causing the critical magnetic shear to be high enough, could the suprathermal electrons have the ability to stabilize the mode. If these conditions is not fully satisfied then double sawtooth, (at a marginal point), partially saturated sawtooth or saturated sawtooth would occur. This is in agreement with the fact that stabilization is achieved for all the partially saturated sawtooth, saturated sawtooth and complete sawtooth, observed in the regime of low density plasmas.

When the ECRH is applied at the low-field side, many of the high-energy electrons are easily trapped in a "banana region" and do not contribute to an enhancement of the current density. As a result, there is a smaller influence on the sawtooth activity.

Local changes in the temperature during ECRH can also cause modifications in the current density profile, which also reduce the shear. It must be noted that the heating effects are generally equally profusely when the ECRH is applied at high-field side or low-field side or axis. As such, they cannot account entirely for the modification of the behaviour of sawtooth, but might contribute to the effect.

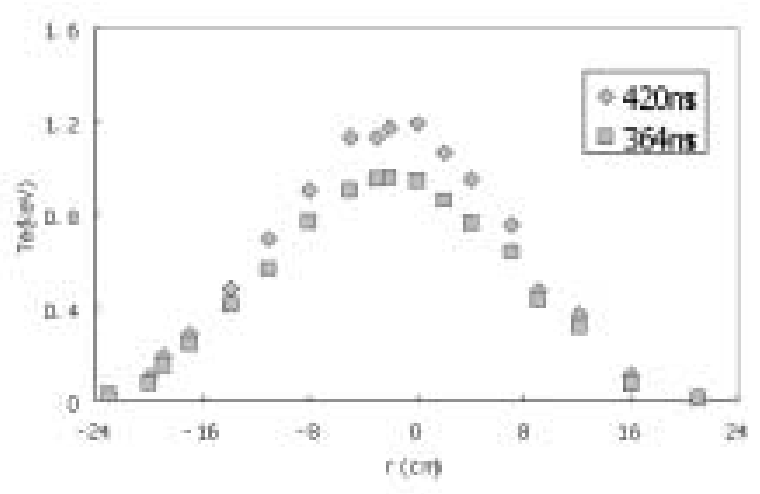

Figure 6. Electron temperature measured by ECE During ECRH. There was a $30 \%$ rise in electron temperature due to $\mathrm{ECH}$, the $T_{e}$ profile did not change much during ECRH heating.

\section{IV.2 Effect of suprathermal electrons on internal kink instability}

It has already been previously reported (DIII-D [1], WT-3 [2], TCV[3]) that it is near the $q=1$ surface that sawtooth oscillations could be stabilized by localized electron-cyclotron-resonance heating. However, our experimental results show that applying the ECRH near the $q=1$ surface is a double-edged sword to the efforts of stabilizing the central MHD instability within that surface. It was found that the effect of ECR in stabilizing the sawtooth activity is much stronger when the ECR deposition location was moved toward the $q=1$ surface within the high-field side, as discussed in IV.1. On the other hand, there is other mechanism [6] through which ECRH has an effect of exciting the internal kink instability. When the off-axis heating location is far from axis and near $q=1$ surface, there are more posibilities for suprathermal electrons being barely-trapped. While the passing electrons play a role to reduce the local shear and tend to stabilize the sawtooth activity, the barely-trapped electrons may enhance or drive an internal kink mode via precessional resonance [6], since the mode propagates poloidally parallel to the ion diamagnetic drift velocity $\left(k_{\theta} / V_{d t}\right)$, and toroidally parallel to the precession velocity $\left\langle V_{\varphi}\right\rangle$ of trapped ions, which is in the same direction as the plasma current. The precessional resonance through Landau damping, between wave and particles, requires that the particles drift along the same poloidal and toroidal direction of the wave. The toroidal drift velocity of particles in Tokamak is given by

$$
\hat{\phi} \cdot \nu_{D}=\nu_{\|}+\frac{\nu_{\|}^{2}+\nu_{\perp}^{2} / 2}{\Omega R_{0}} \frac{B_{P}}{B} \cos \theta
$$

where $\Omega=q B / m$ is the cyclotron frequency. Concerning the population of suprathermal electrons, the passing electrons usually drift in opposite direction due to their opposite electric charges. For the deeply trapped 
electrons on the outboard side $\left(\theta<90^{\circ}\right)$, the pressional direction is opposite to the propagating direction of thr wave. For barely trapped electron (part of its orbit is on the inboard side: $\theta>90^{\circ}$ ), the precessional direction averaged over the entire orbit is parallel to that of the deeply trapped ions because they spend more time on the inboard side and, therefore, this part of the orbit is weighted more. Due to this drift-reversal effect, the barely trapped suprathermal electrons from ECRH heating can resonate with unstable mode and drive the mode in a stronger.

In Fig. 7, the x-ray signals for plasma with different resonance location from the line of sight that passes through the inversion radius are compared. When the cyclotron resonance position is moved to the weak field side, the $m=1$ mode excitation is not observed. Repeated scans show very similar results: the $\mathrm{m}=1$ mode can only be excited when the ECR location is near the $q=1$ surface at the high field side. This indicates that the phenomenon is due to the kinetic effect of suprathermal electrons rather than the change of the plasma pressure.

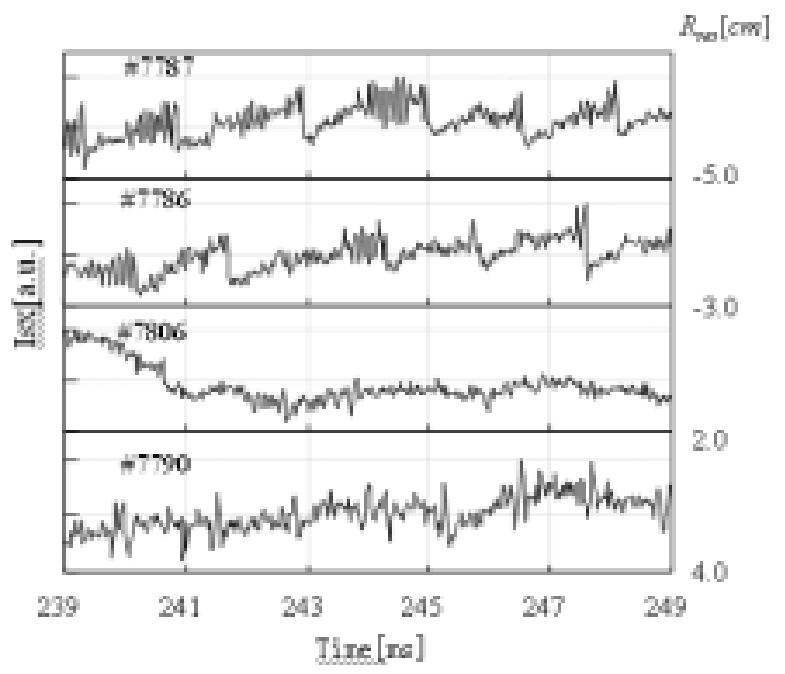

Figure 7. Comparison of the $\mathrm{m}=1$ mode activity at various ECR locations labeled by the distance from the magnetic axis $\left(R_{\text {res }}\right)$. A negative value denotes the high field side of the magnetic axis.

\section{Conclusions}

A significant effect of stabilization/modification of sawteeth activities, due to energetic electrons, have been found in HL-1M ECRH experiments. The stabilization is attributable to a local reduction of the shear near the $q=1$ surface due to energetic electron effects. There is a density threshold below which complete stabilization could be obtained and, above that, the sawtooth would either be saturated, partially saturated or become double sawtooth. While some results suggest that the sawtooth activity may be controlled effectively with ECH at the $q=1$ surface on the high-field side, more careful research show it is near the $q=1$ surface that internal kink instability could be driven by barely-trapped suprathermal electrons during off-axis ECH. The $\mathrm{m}=1$ internal kink instability purely driven by energetic electrons was observed. The energetic trapped electrons were found to play the dominant role in the excitation mechanism. While a qualitative explanation is given, a quantitative explanation for this observation is a challenging research work for the future.

\section{Acknowledgements}

The authors are grateful to Dr. Zhao, J.Y and Professor Yang, J.W for their colaboration in the experiments, to Dr.Yan, L.W and Professor Shi, B.R.for useful discussions, and to Professor Qio, X.M for the continuous encouragement.

\section{References}

[1] W. Pfeiffer, et al., Nucl. Fusion 25, 673 (1985).

[2] K. Hanada, Phys. Rev. Lett. 66, 1974 (1991).

[3] Z.A. Pietrzyk, et al., Nucl. Fusion 39, 587 (1999).

[4] K.L. Wong, Phys. Rev. Lett. 85, 996 (2000).

[5] J.A. Wesson, et al., IAEA-CN-47/E-I-1-1 (1986) 3.

[6] S. Bingren, et al., China nuclear science and technology report CNIC-00963 (1997).

[7] R.A. Cairns "Radiofrequency heating of plasma" Department of mathematical and computational sciences, University of St Andrews, Published under Adam Hilger 1991.

[8] Y. Liu, et al., China nuclear science and technology report CNIC-01279 (1998). 